\title{
Quantum Cascade Lasers in Biomedical Infrared Imaging
}

\author{
Benjamin Bird ${ }^{1 *}$ and Matthew J. Baker ${ }^{2^{*}}$ \\ ${ }^{1}$ Daylight Solutions Inc., 15378 Avenue of Science, San Diego, CA 92128, USA \\ ${ }^{2}$ WestCHEM, Department of Pure and Applied Chemistry, University of Strathclyde, 295 Cathedral Street, \\ Glasgow, G1 1XL, UK
}

*bbird@daylightsolutions.com, matthew.baker@strath.ac.uk,Twitter @DrBenjaminBird, @ChemistryBaker

Keywords: quantum, microscope, infrared, discrete frequency, rapid

Mid-infrared (IR) spectroscopic imaging is a well established fingerprinting technique that permits 'label-free' visualisation of sample microstructure and chemistry. A rapidly expanding field of biospectroscopists are now harnessing this tool to extract potent biochemical information from biomedical samples that can monitor perturbations associated with cellular disease, differentiation, drug resistance and therapeutic response [1]. Fourier Transform infrared (FT-IR) imaging spectrometers, that employ interferometry and profit from the multiplex advantage, have accelerated fundamental research in this emerging field. However, such instrumentation requires the acquisition of spectra from a large bandwidth using a low throughput infrared light source (e.g. globar). More recently, the advent of high throughput broadly-tuneable Quantum Cascade Laser (QCL) based infrared light sources has provided an alternative path to accelerate spectral based microscopy by employing Discrete Frequency IR (DF-IR) imaging [2-5]

The advent and commercialisation of QCLs has allowed the development of new discrete frequency based instruments that exploit the high brightness and narrow bandwidth available with these sources [6-8]. More recently, these laser modules have become available in multiplexed configurations that span the entire biological fingerprint region that compose characteristic vibrational signatures for all biomolecules. This has led to the development of dedicated laser based microscopes that permit interrogation of biomedical samples at sparsely distributed points across the spectrum [2,3]. By targeting frequencies that describe salient information, high-throughput methodologies can be realised that reduce data size and remove computationally expensive Fourier transforms. One distinct advantage of having a highly brilliant source is the ability to use uncooled microbolometer detector systems [3,5]. High-definition IR imaging can also be readily achieved using QCL based microscopes. High numerical aperture (NA), achromatic, refractive based objectives have been developed that permit diffraction-limited resolution from a wide field of view (FOV), ca. 20X larger in size than previously attainable [3]. However, one of the most exciting capabilities of this new type of microscope is the ability to perform real-time discrete frequency chemical imaging that permits 'label-free' spatially resolved molecular contrast with live feedback.

Pioneering work that has taken advantage of these technological breakthroughs has provided proof of concept that biomedical IR imaging can be markedly accelerated. Brightfield microscopy of large biopsy tissue sections or high density tissue microarrays (TMAs) is time prohibitive for FT-IR based microscopes. Mega mosaic DF-IR images have now been acquired in the minute's timescale that provide rapid chemical contrast and microstructure information. Bassan et al. reported the acquisition of a chemical image from a TMA based on the absorbance of the Amide I protein band in 9 minutes [3]. This TMA composed 207 tissue cores covering an area of $2.0 \times 2.4 \mathrm{~cm}^{2}$ at a speed 126 times faster when compared to a FT-IR system, albeit using a single frequency rather than a broadband spectrum. This type of low resolution global chemical view can serve as a rapid tool to pinpoint important FOVs that can be scrutinised at high resolution, similar to the paradigm employed by pathologists. Kröger et al. have demonstrated chemical imaging at diffraction-limited resolution from a FOV $2.8 \times 3.1 \mathrm{~mm}^{2}$ using the spectral range $1030-1090 \mathrm{~cm}^{-1}$ in 5 minutes. By 
utilising clustering methods, differentiation of tissue structures and cell types in mouse jejunum was achieved with a ca. 20 times quicker speed than FT-IR imaging [4]. Yeh et al. have recently exploited QCls that compose the full fingerprint region coupled with a cryogenically cooled camera to record high definition (HD) diffraction-limited mega mosaic images from TMAs in 3-orders of magnitude smaller time per essential spectral frequency when compared to the fastest HD FT-IR bench top systems. The novel microscope also reported a greater resolving power, over FT based systems, when interrogating a standard optical resolution target [2]. The exploitation of high definition DF-IR real-time imaging and sparse frequency collection protocols is further highlighted in Figure 1 (reproduced from [9]) showing microscopic images of a colon thin section analysed via QCL based IR spectroscopy. The figure clearly shows that by use of a few discrete frequenciesaccurate cell type differentiation can be achieved. Colon globlet ( $a-$ green) and epithelial cells ( $b$-red) are provided distinct contrast by use of measurements recorded at two discrete (intensity ratio of 1076 / 1181 $\mathrm{cm}^{-1}$ ) and a limited range ((integral absorbance from $1027-1087 \mathrm{~cm}^{-1}$ ) of frequencies respectively. Each diagnostic application shall require a unique set of frequencies that provide optimal discriminatory power, but customised protocols can now be tailored to remove redundancy and increase throughput of infrared based biomedical imaging.

The future is certainly bright for QCL based IR microscopy and biomedical research. Significant improvements in signal-to-noise, speed, resolution and instrument footprint can be achieved and improved upon as the technology matures. The realisation of adjunctive tools for clinical researchers does not seem as far off as it once did.

\section{References}

[1] Baker MJ et al. (2014), Using Fourier transform IR spectroscopy to analyze biological materials, Nature Protocols, 9(8), 1771-1791

[2] Yeh K, Kenkel S, Liu JN, Bhargava R (2015) Fast Infrared Chemical Imaging with a Quantum Cascade Laser Anal. Chem. 87(1), 485-493

[3] Bassan P, Weida MJ, Rowlette J, Gardner P (2014), Large scale infrared imaging of tissue micro arrays (TMAs) using a tunable Quantum Cascade Laser (QCL) based microscope, Analyst, 139, 38563859

[4] Kröger-Lui N, Egl A, Engel M, Gretx N, Haase K, Herpich I, Kränzlin B, Neudecker S, Pucci A, Schönhals A, Vogt J, Petrich W (2014), Quantum cascade laser-based hypersepctral imaging of biological tissue, Journal of Biomedical Optics, 19(11), 111607

[5] Kole MR, Reddy RK, Schulmerich MV, Gelber MK, Bhargava R (2012), Discrete frequency infrared microspectroscopy and imaging with a tunable quantum cascade laser, Anal. Chem., 84(23), 1036610372

[6] Brandsetter M, Koch C, Genner A, Lendl B (2013), Measures for optimizing pulsed EC-QC laser spectroscopy of liquids and application to multi-analyte blood analysis, Proc SPIE 899s Quantum Sensing and Nanophotonic Devices XI, 89931U

[7] Worle K, Seichter F, Wilk A, Armacost C, Day T, Godejohann M, Wachter U, Vogt J, Radermacher P, Mizaikoff B (2013), Anal. Chem. 85, 2697-2702

[8] Liakatt S, Bors KA, Xu L, Woods, CM, Doyle J, Gmachi CF (2014), Noninvasice in vivo glucose sensing on human subjects using mid-infrared light, Biomedical Optics Express, 5(7), 2397-2404

[9] Kröger-Lui N, Gretz N, Hasse K, Kränzlin B, Neudecker S, Pucci A, Regenschiet A, Schönhals A, Petrich W (2015), Analyst, 2014, 2086-2092 
Microscopic IR images of colon thin sections (a) represents the ratio of absorbances at $1076 \mathrm{~cm}^{-1}$ and $1181 \mathrm{~cm}^{-1}$ and (b) represents the integral absorbance over the $1027 \mathrm{~cm}^{-1}-1087 \mathrm{~cm}^{-1}$ spectral range. Reproduced from [9] with permission from the Royal Society of Chemistry
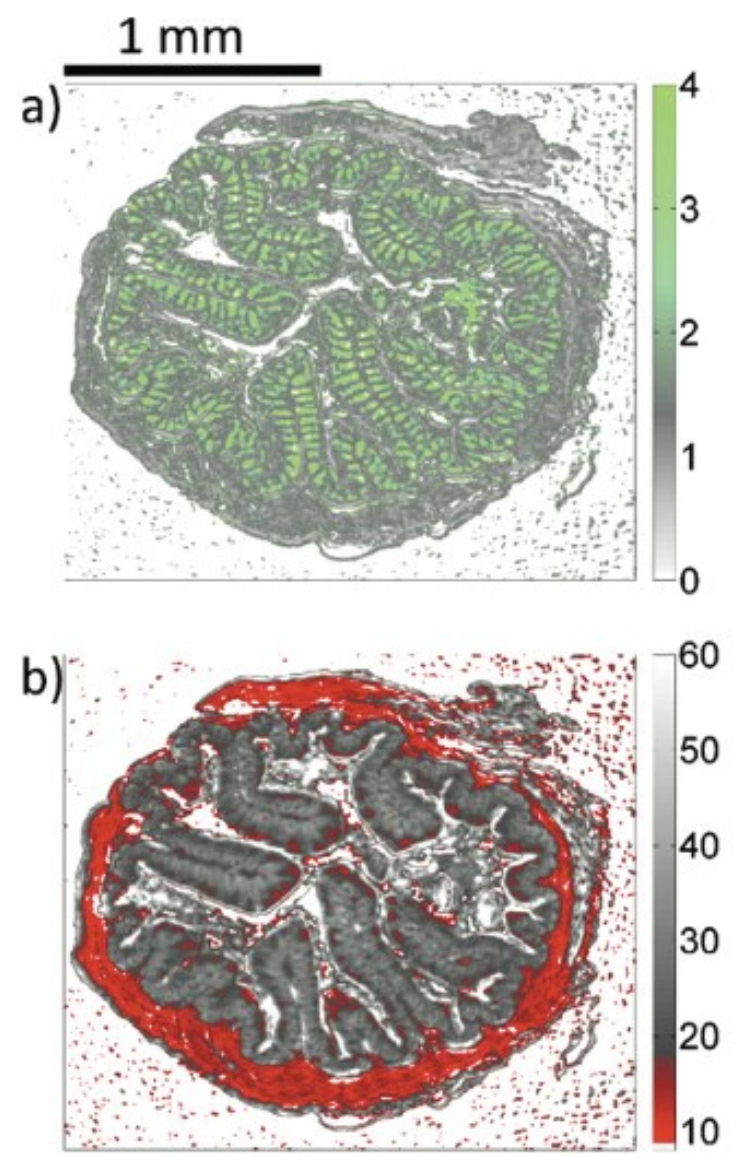